\title{
Conversión de las explotaciones de vacuno de leche a la producción de leche $A 2$ ante una posible demanda del mercado: posibilidades e implicaciones
}

\author{
L. Alfonso*, O. Urrutia y J.A. Mendizabal
}

Escuela Técnica Superior de Ingenieros Agrónomos, IS-FOOD Research Institute, Universidad Pública de Navarra, Campus Arrosadia, Pamplona, España

\section{Resumen}

El mercado de la leche de vaca, tras la desaparición de las cuotas lácteas, está provocando una importante restructuración en el sector. Una de las alternativas que están desarrollando, fundamentalmente las pequeñas explotaciones familiares, es la diferenciación de sus productos. En ese contexto, ha aparecido un tipo diferenciado de leche conocida como leche A2. Esta se caracteriza por estar libre de la variante $A 1$ de la proteína $\beta$-caseína que, debido a una mayor producción de $\beta$-casomorfina-7 tras la digestión, puede dar lugar a intolerancias y afecciones gastrointestinales. En este trabajo se analizan las posibilidades e implicaciones de una selección a favor de la $\beta$-caseína A2 en el caso concreto de las explotaciones familiares de Guipúzcoa. Los resultados obtenidos a partir de 1868 hembras genotipadas muestran que la frecuencia del alelo que codifica la proteína A2 es 0,55, y no se han encontrado asociaciones desfavorables entre el alelo A2 y el valor genético para caracteres productivos, funcionales y morfológicos, ni con otros genes ni haplotipos. Bajo esta situación de partida, y empleando exclusivamente toros A2A2, la utilización o no de semen sexado, junto con las diferentes ratios de reposición que se apliquen, determinarán el periodo y el coste económico de la conversión de los rebaños a la producción de leche A2. En un escenario de costes razonables esta se estima entre los 8 y los 15 años, pudiendo llegar

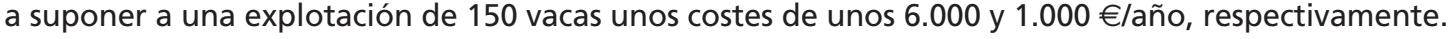

Palabras clave: Leche, $\beta$-caseína, genotipado, selección, reconversión, vacuno.

Conversion to A2 milk production with regard to a possible market demand for dairy farms: possibilities and implications

\begin{abstract}
Bovine dairy market, after the abolition of milk quotas, is causing dairy sector restructuration. Product differentiation is one of the strategies that are implementing dairy farms, mainly small family farms. In this context, a new type of differentiated cow s milk has appeared known commercially as A2 milk. This type of milk is devoid of A1 $\beta$-casein protein, which can cause intolerance and gastrointestinal discomfort due to higher $\beta$-casomorphin-7 release after digestion. In the present work, the possibilities and implications of a selection in favor of A2 $\beta$-casein were analysed in family farms in Guipúzcoa (Spain). The results obtained from 1868 genotyped females showed that the frequency of allele coding the $A 2$
\end{abstract}

* Autor para correspondencia: leo.alfonso@unavarra.es

Cita del artículo: Alfonso L, Urrutia O, Mendizabal JA (2019). Conversión de las explotaciones de vacuno de leche a la producción de leche A2 ante una posible demanda del mercado: posibilidades e implicaciones. ITEA-Información Técnica Económica Agraria 115(3): 231-251. https://doi.org/10.12706/itea.2019.001 
protein was 0.55 and no unfavorable associations were found between $\mathrm{A} 2$ allele and the breeding values for production, functional and morphological traits nor other genes or haplotypes. Under this scenario, and using only A2A2 bulls, the use or not of sexed semen, together with different replacement rates that are applied, will determine the period and the economic cost of herd conversion to A2 milk production. In a reasonable cost strategy, the estimated conversion period is between 8 and 15 years, which, in a 150-cow farm, could imply an approximate cost between 6,000 and 1,000 €/year, respectively.

Keywords: Milk, $\beta$-casein, genetic testing, herd selection, conversion, cattle.

\section{Introducción}

El mercado de la leche de vaca sufre una creciente globalización que obliga a los ganaderos a aumentar continuamente el tamaño de las explotaciones, reducir constantemente costes, dejar la actividad, o desacoplarse del mercado mundial mediante la diferenciación de productos. En ese contexto, la empresa neozelandesa 'The a2 Milk Company' lanzó al mercado hace unos años la leche A2 (a2 Milk $\left.{ }^{\mathrm{TM}}\right)$. Con una fuerte inversión en marketing, defensa de la propiedad intelectual e I+D (8,4\% del total de ingresos, en el año 2017, equivalente a unos 27 millones de euros) ha conseguido estar presente en otros países como Australia, Estados Unidos, Reino Unido y China (Babidge, 2017).

La leche A2 se caracteriza por estar libre de la variante $A 1$ de la $\beta$-caseína, proteína que representa sobre el $30 \%$ de la proteína de la leche de vaca (Brooke-Taylor et al., 2017). Existen dos variantes fundamentales de la $\beta$-caseína, la A1 y la A2. La variante A2 es la habitual en todo tipo de leche, pero en las poblaciones de vacuno Holstein-Frisón la variante A1 también se encuentra con una importante frecuencia (Kamiński et al., 2007; Gödert et al., 2017; Massella et al., 2017). La variante A1 se diferencia de la $\mathrm{A} 2$ en un único cambio en la cadena de aminoácidos (sustituye Prolina por Histidina en la posición 67) que se ha considerado que puede afectar a las propiedades de la leche en relación a la salud humana (Asledottir et al., 2017). Inicialmente se especuló, aunque no parece existir asociación, que la variante A1 facilitaba los procesos inmunológicos que conducen a la diabetes tipo I y que era un factor de riesgo para sufrir enfermedades coronarias (Truswell, 2005). Actualmente, se considera que la variante A1 puede estar asociada con la intolerancia a la leche y algunos problemas gastrointestinales, debido a una mayor producción de $\beta$-casomorfina-7 tras la digestión gastrointestinal (Asledottir et al., 2017). No obstante, el número de ensayos clínicos realizados y publicados hasta la fecha en humanos -todos con participación de 'The a2 Milk Company'- es escaso (Ho et al., 2014; Jianquin et al., 2016; He et al., 2017), y pese a sugerir que el consumo de leche A2 puede atenuar los síntomas gastrointestinales de la intolerancia a la leche, no se pueden considerar concluyentes (Brooke-Taylor et al., 2017).

De todas formas, no es objeto de este trabajo dilucidar sobre los posibles efectos positivos de la leche A2 sobre la salud humana. El objetivo es analizar las posibilidades y las implicaciones de conversión de las explotaciones de vacuno de leche a la producción de leche A2 ante la posibilidad de que aparezcan nuevas demandas de mercado.

La demanda de leche A2 por parte de la industria puede ser una oportunidad, pero a la vez una amenaza para los pequeños productores de vacuno de leche que mantienen una estructura familiar de producción. A corto plazo, para las pequeñas explotaciones familiares que se enfrentan a los riesgos de la reestructuración del sector tras la desaparición de las cuotas lácteas, la producción de le- 
che A2 puede suponer una oportunidad para encontrar un nicho de mercado diferenciado. Ya existen algunos ejemplos a nivel estatal ${ }^{1}$. A medio plazo, disponer de rebaños libres del tipo A1 puede significar estar preparados ante una posible demanda de la industria, no ya para obtener un mayor precio por la venta de leche sino para poder asegurar su recogida y venta. No obstante, hay que tener en cuenta que desde hace tiempo se han descrito asociaciones entre los polimorfismos de la $\beta$-caseína y algunos caracteres de interés productivo en algunas poblaciones de vacuno (Lin et al., 1989; Velmala et al., 1995), que en caso de existir afectarían al nivel genético y productivo de las explotaciones que realizaran una conversión a leche A2.

Este trabajo analiza las posibilidades e implicaciones de una selección a favor de la $\beta$-caseína tipo A2 para valorar el interés de empezar a trabajar en la selección de vacas A2A2 en el caso concreto de las explotaciones familiares de Guipúzcoa. Concretamente, el objetivo es: 1) estimar frecuencias alélicas y genotípicas; 2 ) analizar la asociación entre el genotipo de las hembras y su valor genético para caracteres de interés productivo; 3 ) analizar las implicaciones del uso exclusivo de toros A2A2; y 4) determinar la duración y el coste de la conversión bajo distintas estrategias.

\section{Material y métodos}

\section{Material}

Se analizaron los datos disponibles de 27 explotaciones de GIFE (Gipuzkoako Frisoi Elkartea/Asociación Frisona de Guipúzcoa) que participan en un programa de evaluación genómica de novillas iniciado en el año 2015 (Frisona Española, 2017). La mayor parte son pequeñas explotaciones familiares con menos de 100 vacas en producción (Tabla 1) y representan aproximadamente una cuarta parte de los socios de GIFE. En el momento en que se tomaron los datos, las 27 explotaciones englobaban 4216 hembras, alrededor del $30 \%$ del censo de Guipúzcoa, de las cuales 1868 habían sido genotipadas mediante el chip de baja densidad EuroG10K (Jiménez, 2015a) utilizando el servicio de evaluación genómica de hembras de CONAFE.

Además de marcadores SNP para el cálculo del valor genómico de diversos caracteres, el chip EuroG10K incluye marcadores específicos de enfermedades genéticas, de haplotipos relacionados con la fertilidad, del color de la capa y de proteínas lácteas, entre ellas la $\beta$-caseína. Se dispuso así de información del polimorfismo de la $\beta$-caseína de las hembras genotipadas, diferenciando entre el grupo de polimorfismos A1 (alelos A1, B, C, D, F y G) y el resto de polimorfismos que no presentan variación en la posición 67 de la cadena de aminoácidos y que se engloban dentro del grupo de polimorfismos $A 2$ de la $\beta$-caseína (alelos A2, A3, E, H1, H2 y H3) (Mencarini et al., 2013). También se dispuso de la información del valor genómico predicho de las 1868 hembras genotipadas para los caracteres recogidos en la Tabla 2, y la información genotípica para los genes y haplotipos que se indican en la Tabla 3. Adicionalmente, se empleó la información del catálogo de toros probados y en prueba de Aberekin, centro de inseminación artificial que da servicio a los ganaderos de la zona, aunque en algunos casos estos también se proveen de semen comercializado por otras empresas. Consultado en abril de 2018, el catálogo tenía 17 toros probados A2A2 (16\% del total de toros probados) y 33 toros genómicos en prueba A2A2 (44\% del total de toros en prueba).

1. p.ej. Granja Armengol V Premio 'CCNIEC/Marc Viader' a la innovación en productos alimenticios. 
Tabla 1. Descriptivos básicos de las explotaciones y los animales analizados.

Table 1. Basic descriptives of farms and analysed animals.

\begin{tabular}{|c|c|c|}
\hline \multicolumn{3}{|l|}{$\mathrm{N}^{\circ}$ de explotaciones según tamaño } \\
\hline total & \multicolumn{2}{|c|}{27} \\
\hline con $<100$ vacas & \multicolumn{2}{|c|}{19} \\
\hline con $100-200$ vacas & \multicolumn{2}{|c|}{6} \\
\hline \multirow[t]{2}{*}{ con $>200$ vacas } & \multicolumn{2}{|c|}{2} \\
\hline & Media & Rango \\
\hline Edad media de las vacas ${ }^{1}$ (años) & 3,84 & $2,40-4,85$ \\
\hline Reposición anual estimada² (\%) & 38 & $14-50$ \\
\hline \multicolumn{3}{|l|}{$\mathrm{N}^{\circ}$ de animales } \\
\hline total & \multicolumn{2}{|c|}{4216} \\
\hline terneras & \multicolumn{2}{|c|}{881} \\
\hline novillas & \multicolumn{2}{|c|}{935} \\
\hline vacas & \multicolumn{2}{|c|}{2400} \\
\hline \multicolumn{3}{|l|}{$\mathrm{N}^{\circ}$ de animales genotipados } \\
\hline total & \multicolumn{2}{|c|}{1868} \\
\hline terneras & \multicolumn{2}{|c|}{673} \\
\hline novillas & \multicolumn{2}{|c|}{713} \\
\hline vacas & \multicolumn{2}{|c|}{484} \\
\hline $\mathrm{N}^{\circ}$ de padres de los animales genotipados & \multicolumn{2}{|c|}{262} \\
\hline $\mathrm{N}^{\circ}$ de abuelos maternos de los animales genotipados & \multicolumn{2}{|c|}{402} \\
\hline
\end{tabular}

${ }^{1}$ Hembras de más de 2 años.

${ }^{2}$ Calculada como la mitad del número de terneras y novillas de recría por cada 100 vacas.

\section{Métodos}

Estimación de frecuencias alélicas y análisis de asociación con otros caracteres

Las frecuencias alélicas para los polimorfismos $A 1$ y $A 2$ de la $\beta$-caseína se estimaron por conteo a partir de la información genotípica, contrastando mediante prueba $\chi^{2}$ el equilibrio de Hardy-Weinberg. La estimación sólo se realizó para las hembras, al no tener disponible la información genotípica de todos los machos utilizados.
La asociación estadística entre los polimorfismos $A 1$ y $A 2$ de la $\beta$-caseína y los distintos caracteres con valores genómicos predichos (Tabla 2) se analizó mediante análisis de varianza simple, sin tener en cuenta la fiabilidad de los valores predichos dada su escasa variabilidad al tratarse de valores genómicos. La asociación con otros genes y haplotipos (Tabla 3 ) se analizó mediante prueba $\chi^{2}$. Los análisis se realizaron utilizando $\mathrm{R}$ ( $\mathrm{R}$ Core Team, v.3.3.2). 
Tabla 2. Caracteres con valores genómicos predichos para las hembras analizadas $(n=1868)$. Table 2. Traits with predicted genomic values for the analysed females $(n=1868)$.

\begin{tabular}{|c|c|c|}
\hline Grupo & Carácter & Abreviatura \\
\hline \multirow[t]{5}{*}{ Productivos } & Kilogramos de leche & $\mathrm{KL}$ \\
\hline & Porcentaje de grasa & $\% \mathrm{G}$ \\
\hline & Kilogramos de grasa & KG \\
\hline & Porcentaje de proteína & $\% \mathrm{P}$ \\
\hline & Kilogramos de proteína & $\mathrm{KP}$ \\
\hline \multirow[t]{4}{*}{ Funcionales } & Recuento de células somáticas & RCS \\
\hline & Longevidad funcional & LON \\
\hline & Días abiertos & DA \\
\hline & Velocidad ordeño & vo \\
\hline \multirow[t]{18}{*}{ Morfológicos } & Estatura & EST \\
\hline & Anchura de pecho & ANPE \\
\hline & Profundidad corporal & PC \\
\hline & Anchura de grupa & ANCG \\
\hline & Ángulo de grupa & ANGG \\
\hline & Angulosidad & ANGU \\
\hline & Vista lateral de patas traseras & VLP \\
\hline & Vista posterior de patas traseras & VPP \\
\hline & Ángulo podal & AP \\
\hline & Miembros y aplomos & MA \\
\hline & Inserción anterior & IAN \\
\hline & Altura inserción posterior & AIP \\
\hline & Ligamento suspensor & LIG \\
\hline & Profundidad de ubre & PU \\
\hline & Colocación de pezones anteriores & CPA \\
\hline & Colocación de pezones posteriores & CPP \\
\hline & Longitud de pezones anteriores & LPA \\
\hline & Movilidad & MOV \\
\hline \multirow[t]{7}{*}{ Sintéticos } & Índice de mérito genético total & $\mathrm{ICO}$ \\
\hline & Componente de producción del ICO & ICOp \\
\hline & Componente morfológico del ICO & ICOt \\
\hline & Índice de Capacidad & ICAP \\
\hline & Índice de Patas y Pies & IPP \\
\hline & Índice Compuesto de Ubre & ICU \\
\hline & Índice Global de Tipo & IGT \\
\hline
\end{tabular}


Tabla 3. Información genotípica disponible de genes y haplotipos. Table 3. Available genotypic information of genes and haplotypes.

\begin{tabular}{lcc}
\hline Grupo & Gen/Haplotipo & $\mathrm{n}$ \\
\hline Proteínas lácteas & $\beta$-Caseína & 1868 \\
$\alpha s_{1}$-Caseína & 62 \\
$\alpha s_{2}$-Caseína & 62 \\
$\beta$-LactoGlobulina & 1865 \\
Kenes recesivos & DUMPS & 1747 \\
& BLAD & 1868 \\
Mulefoot & 1868 \\
CVM & 1868 \\
Brachyspina & 1868 \\
Citrulinemia & 706 \\
HH1 & 1868 \\
HH2 & 1868 \\
HH3 & 1868 \\
HH4 & 1868 \\
HH5 & 1868 \\
HDC & 1868 \\
\hline
\end{tabular}

Análisis de distintas estrategias de conversión a leche $A 2$ e implicaciones del uso de toros A2A2

Se simularon cuatro escenarios de reconversión de rebaños a la producción de leche A2:

Escenario 1: - utilización exclusiva de toros A2A2

Escenario 2: - utilización exclusiva de toros A2A2

- utilización de semen sexado

Escenario 3: - utilización exclusiva de toros A2A2
- utilización de semen sexado

- aumento de la reposición de vacas $\mathrm{A} 1 \mathrm{~A} 1$

Escenario 4: - utilización exclusiva de toros A2A2

- utilización de semen sexado

- aumento de la reposición de vacas A1A1

- aumento de la reposición de vacas $A 1 A 2$

Para cada escenario se consideró un periodo de 15 años a partir de la toma de decisión de la conversión, asumiendo que el proceso de 
conversión se alcanzaba cuando el porcentaje de vacas A2A2 era superior al $97 \%$, de forma semejante al trabajo de Gödert et al. (2017). La Tabla 4 recoge los cálculos realizados para determinar cada año el número de vacas A2A2 presentes en el rebaño. Se consideraron distintas frecuencias iniciales del polimorfismo $A 2$ en la población $(0,55$ y 0,35), distintas tasas de reposición media anual involuntaria $(25,38$ y $50 \%)$, distintas tasas de reposición media anual voluntaria (0 y $37 \%$ ), una sex-ratio de 0,5 y 1 (para el semen no sexado y sexado, respectivamente), un intervalo entre partos, estimado en las explotaciones, de 420 días y una tasa de descarte del $10 \%$ de terneras y novillas durante la recría.

Para valorar el retraso genético que podría suponer el empleo de exclusivamente toros homozigotos A2 durante la conversión de una explotación a leche $A 2$ se compararon las medias de los valores genéticos de los toros A2A2 frente al resto de toros disponibles en el centro de inseminación artificial considerado. El análisis se realizó mediante comparación simple de medias para los toros probados y en prueba por separado, dadas las diferencias en la fiabilidad de sus valores predichos entre ambos grupos.

Finalmente, se aproximó el coste económico de la conversión calculando por separado los costes de genotipado, la compra de semen sexado y la reposición voluntaria (el aumento de la reposición de vacas A1A1 y A1A2), para cada uno de los años del periodo estimado de conversión de cada escenario. Se asumió un coste de genotipado de $30 €$ por animal, un extracoste de la dosis seminal de semen sexado de $15 €$ (que asumiendo 2,5 inseminaciones por parto suponía un coste total del uso de semen sexado de $37,5 €$ por lactación), y un coste de recría de $1.800 €$ por novilla. El primer año, el coste de genotipado se imputó a todas las vacas presentes en la explotación. Los siguientes años, y hasta alcanzar el mínimo de un $97 \%$ de vacas A2A2, el coste sólo se imputó a las terneras nacidas de vacas heterozigotas A1A2. El coste del semen sexado se imputó desde el primer año, y el coste de la reposición voluntaria desde el segundo, momento a partir del cual se dispone de novillas de reposición genotipadas e hijas de toros A2A2.

\section{Resultados y discusión}

\section{Frecuencias alélicas y genotípicas}

La frecuencia del alelo que codifica la proteína $A 2$ en los animales genotipados es de 0,55 semejante a la estimada en otras poblaciones europeas actuales de Holstein (Massella et al., 2017) y a la estimada hace décadas en las poblaciones de Holstein estadounidenses (van Eenennaam y Medrano, 1991). No obstante, es superior a la estimada en las poblaciones de Holstein canadiense (Ng-Kwai-Hang et al., 1984) y neozelandés (Morris et al., 2005), y en la población española de Holstein-Frisón de la década de los ochenta (Tejedor, 1986), en las que el alelo que codifica la proteína $A 1$ era el más frecuente. Las frecuencias genotípicas estimadas han sido 0,31 para $A 2 A 2,0,48$ para A1A2 y 0,21 para A1A1, indicando que la población está en aparente equilibrio de HardyWeinberg $(p<0,05)$ para la $\beta$-caseína.

Las variaciones de la frecuencia del alelo que codifica la proteína A2 entre las explotaciones analizadas fueron importantes, posiblemente debido únicamente a su pequeño tamaño, oscilando entre 0,35 y 0,67 , lo que indica que las explotaciones se encuentran en distinta situación de partida para la conversión a producción de leche $A 2$, con posibles implicaciones en la duración y el coste de la conversión. 


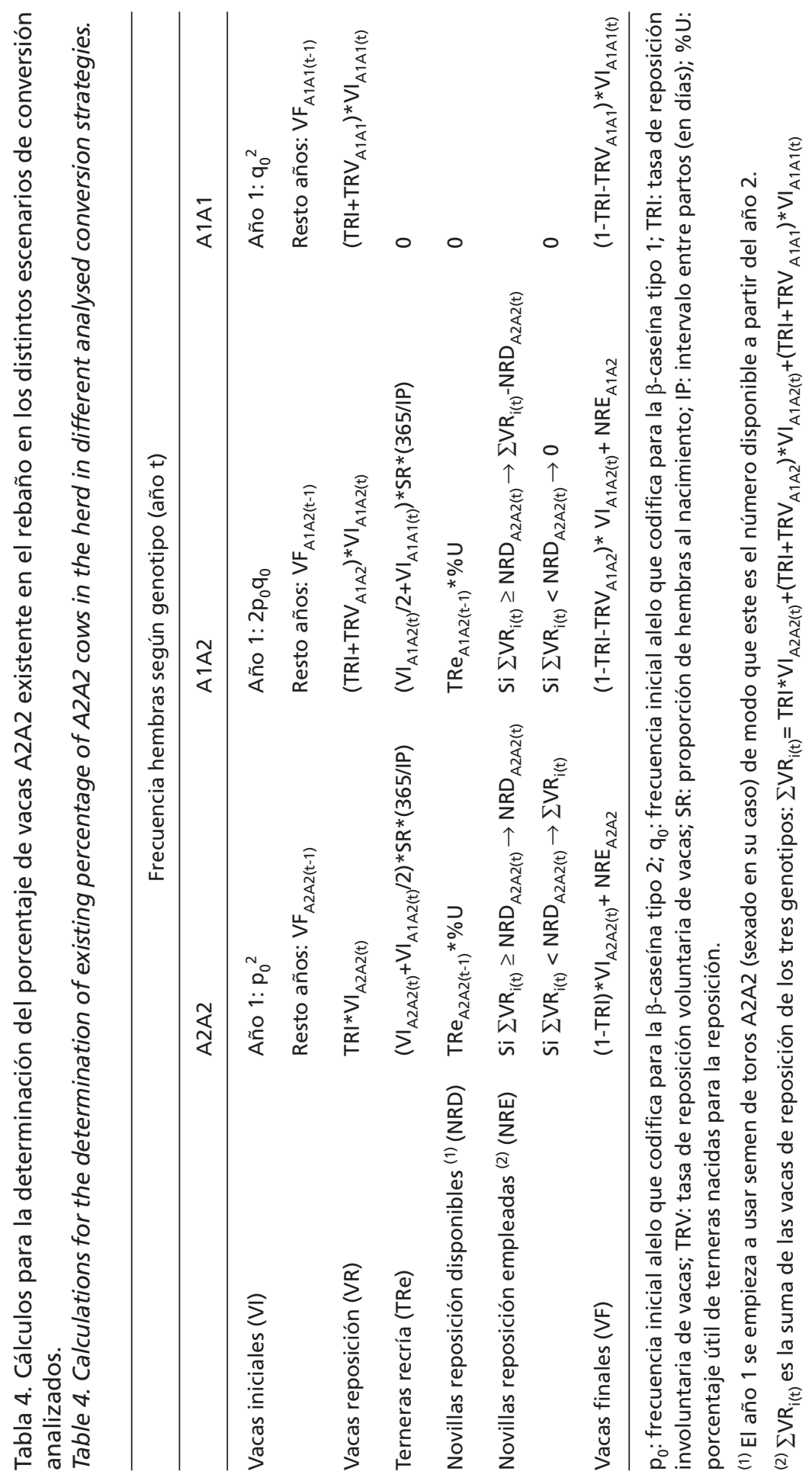




\section{Asociación entre el genotipo para $\beta$-caseína $y$ otros caracteres}

Como se muestra en la Tabla 5 en las explotaciones analizadas se encontró la existencia de asociación estadística entre el genotipo para $\beta$-caseína y el valor genómico para $\mathrm{KL}$, $\mathrm{KP}, \% \mathrm{G}$ e ICOp. Esa asociación indica que la selección de los animales A2A2 aumentaría ligeramente el valor genómico medio para kilogramos de leche y proteína (alrededor de un $9 \%$ ), pero disminuiría de forma importante (sobre un $70 \%$ ) el porcentaje de grasa. No obstante, esa disminución no afecta a los valores del ICO, ni tampoco afecta negativamente a los valores de la componente de producción del ICO, dado que el ICO no considera el porcentaje de grasa (incluye KL, KG, KP).

Estos resultados son coincidentes con las asociaciones referidas en otras poblaciones Holstein; el alelo que codifica la proteína A2 se asocia a un aumento de la cantidad de leche y proteína y un menor contenido de grasa, lo que le llevó a ser considerado como un mar- cador para la selección de cantidad de proteína y leche (Lin et al., 1989; Morris et al., 2005; Olenski et al., 2010). Sin embargo en otros trabajos, el alelo de la proteína A2 por sí sólo no mostraba relación con la producción de leche y proteína, presentando únicamente relación en combinación con el gen de la א-caseína (Ojala et al., 1996).

Respecto a los caracteres funcionales, la selección de exclusivamente novillas A2A2 no afectaría, de acuerdo a los resultados de la Tabla 6, de forma importante. A lo sumo cabría esperar una muy ligera reducción $(\leq 1 \%)$, favorable del recuento de células somáticas y desfavorable de la longevidad funcional. Morris et al. (2005) encontraron un mayor recuento de células somáticas en el genotipo $A 1 A 2$ que en el $A 1 A 1$, aunque el porcentaje de vacas con más de 400.000 células $/ \mathrm{ml}$ no variaba significativamente. Por su parte, Lin et al. (1989) no encontraron relación entre el genotipo para $\beta$-caseína y la longevidad de las vacas al analizarla durante los cinco primeros años de vida.

Tabla 5. Valores genómicos medios de los caracteres de producción para los genotipos de $\beta$-caseína $(n=1868)$.

Table 5. Mean genomic values of productions traits for $\beta$-casein genotypes $(n=1868)$.

\begin{tabular}{lccccc}
\hline Carácter $^{1}$ & \multicolumn{3}{c}{ Genotipo } & \multicolumn{2}{c}{ Significación } \\
\cline { 2 - 4 } & A1A1 & A1A2 & A2A2 & & $* * *$ \\
KL & $623,2^{\mathrm{a}}$ & $630,2^{\mathrm{a}}$ & $712,2^{\mathrm{b}}$ & $<0,001$ & $* * *$ \\
KG & $0,060^{\mathrm{a}}$ & $0,040^{\mathrm{a}}$ & $0,011^{\mathrm{b}}$ & $<0,001$ & \\
$\% \mathrm{P}$ & 27,8 & 26,0 & 26,0 & 0,173 & \\
KP & 0,011 & 0,006 & 0,010 & 0,585 & $* * *$ \\
ICO & $21,3^{\mathrm{a}}$ & $21,0^{\mathrm{a}}$ & $24,0^{\mathrm{b}}$ & $<0,0001$ & \\
ICOp & 2711,3 & 2652,4 & 2680,4 & 0,065 & $* * *$ \\
\hline
\end{tabular}

1 Ver el significado de las abreviaturas de los caracteres en la Tabla 2.

Distintas letras entre columnas indican diferencias significativas $(p<0,05)$. 
Tabla 6. Valores genómicos medios de los caracteres funcionales para los genotipos de $\beta$-caseína $(n=$ 1868).

Table 5. Mean genomic values of functional traits for $\beta$-casein genotypes $(n=1868)$.

\begin{tabular}{lcccccc}
\hline \multirow{2}{*}{ Carácter $^{1}$} & \multicolumn{3}{c}{ Genotipo } & \multicolumn{2}{c}{ Significación } \\
\cline { 2 - 4 } & A1A1 & A1A2 & A2A2 & & $*$ \\
\hline RCS & $105,0^{\mathrm{a}}$ & $104,1^{\mathrm{ab}}$ & $103,7^{\mathrm{b}}$ & & 0,033 & $*$ \\
LON & $110,7^{\mathrm{a}}$ & $109,5^{\mathrm{b}}$ & $108,2^{\mathrm{c}}$ & & $<0,0001$ & $* * *$ \\
DA & 100,4 & 100,3 & 99,7 & & 0,331 & \\
VO & 31,8 & 31,8 & 29,6 & 0,635 & \\
\hline
\end{tabular}

${ }^{1}$ Ver el significado de las abreviaturas de los caracteres en la Tabla 2.

Distintas letras entre columnas indican diferencias significativas $(p<0,05)$.

En relación a otras proteínas de la leche, no se encontró relación entre $\beta$-caseína y $\beta$-lactoglobulina $(p>0,05)$. Las escasas hembras con información para las caseínas $\alpha s_{1}$ y $\alpha s_{2}$ eran homozigotas para los alelos $B$ y A respectivamente. Por contra, sí se observó una relación con la $\kappa$-caseína $(p<0,05)$, debida a que los animales A2A2 no presentaban, a diferencia de los animales A1A1, el alelo E de la א-caseína (Tabla 7). En este sentido, Velmala et al. (1995) encontraron en una población de Finnish Ayrshire una frecuencia muy baja del haplotipo A2/E frente al haplotipo A1/E. En cualquier caso, esta asociación no supondría un efecto negativo de la selección de animales $\mathrm{A} 2 \mathrm{~A} 2$, probablemente ocurriría al contrario por el efecto no deseable del alelo E sobre la fabricación de queso (Hallén et al., 2007).

Tabla 7. Frecuencias (\%) de los genotipos de $\beta$-caseína para cada uno de los genotipos de $\kappa$-caseína $(n=1747)$.

Table 7. Frequencies (\%) of $\beta$-casein genotypes for different $\kappa$-casein genotypes $(n=1747)$.

\begin{tabular}{lrrr}
\hline \multirow{2}{*}{ K-caseína } & \multicolumn{3}{c}{$\beta$-caseína } \\
\cline { 2 - 4 } & A1A1 & A1A2 & A2A2 \\
\hline AA & 6,1 & 38,8 & 55,1 \\
AB & 11,5 & 39,7 & 48,8 \\
AE & 28,6 & 71,4 & 0 \\
BB & 21,8 & 53,5 & 24,7 \\
BE & 43,1 & 56,9 & 0 \\
EE & 100 & 0 & 0 \\
\hline
\end{tabular}


Tampoco se encontró asociación entre $\beta$-caseína y las frecuencias de los genes recesivos de BLAD, CVM, DUMPS, Mulefoot y Citrulinemia, y las de los haplotipos $\mathrm{HH} 1, \mathrm{HH}$, $\mathrm{HH} 3, \mathrm{HH} 4, \mathrm{HH} 5(p>0,05)$. Sólo se encontraron asociaciones significativas $(p<0,05)$ con Brachyspina y el haplotipo HDC. Analizando el genotipo de las hijas de los toros más representados en las hembras genotipadas se observó que la asociación entre $\beta$-caseína y Brachyspina podía ser debida al uso de un toro (Etxalde Biarriz), aparentemente portador de Brachyspina y homozigoto A1 para $\beta$ caseína; la asociación desaparece $(p=0,62)$ cuando se eliminan sus hijas del análisis de asociación. De forma parecida, la asociación con HDC dejaba de ser significativa $(p=0,20)$ si se eliminaban del análisis las hijas de un toro (Alh Regan Mizuno), portador del alelo que codifica la proteína A2 y que presenta una elevada proporción de hijas portadoras del haplotipo HDC. En la genealogía de Alh Regan Mizuno aparece Goldwyn y por lo tanto podría ser portador de la mutación, causante de la mortalidad temprana de terneros, que apareció en Storm (Jiménez, 2015b). En resumen, no cabe esperar un aumento de la frecuencia de alelos recesivos ni haplotipos desfavorables por selección de hembras A2A2 si se diseñan adecuadamente los apareamientos.

Finalmente, respecto a las características de tipo, se observaron bastantes variaciones entre genotipos de $\beta$-caseína (Tabla 8). Para los índices sintéticos de tipo las hembras A2A2 presentaron menores valores para IPP e ICU y consecuentemente para la componente morfológica del ICO (determinada sólo por ambos índices). La diferencia fue más importante para IPP $(>10 \%)$ que para ICU $(<5 \%)$ (Tabla 7). Para IPP todos sus componentes (MA, VPP, AP y VLP) fueron significativamente peores para el genotipo $A 2 A 2$, especialmente para VLP, con patas posteriores más curvas en las hembras de genotipo A2A2 (Tabla 8). Tam- bién son significativamente menores los componentes del ICU aunque en este caso no para todos dado que no hay diferencias para IAN y CPA, y siendo las diferencias especialmente importantes para LPA, presentando una longitud de pezones anteriores menos adecuada las hembras A2A2 (Tabla 8). Como consecuencia de las diferencias observadas en los caracteres de tipo que definen IPP e ICU, también se observó un ligero menor valor medio para IGT en las hembras A2A2. Finalmente, se puede señalar que las hembras A2A2 presentaron un valor menor para movilidad (pequeño: $<8 \%$ ) y para ángulo de grupa, en este último caso de mayor magnitud (>50\%).

Aunque también se han referido diferencias morfológicas para caracteres de patas y ubres en vacuno de leche entre genotipos (Potočnik et al., 2016) se hace difícil interpretar este tipo de asociaciones sin tener en cuenta la morfología preferentemente seleccionadas en cada explotación en función de sus características o gustos del ganadero.

\section{Duración del periodo de reconversión $y$ coste económico}

La Figura 1 recoge la evolución del porcentaje de vacas A2A2 en los distintos escenarios encaminados a la obtención de rebaños A2A2 analizados: 1) tasa de reposición habitual - semen sin sexar; 2) tasa de reposición habitual - semen sexado; 3 ) doble de la tasa de reposición de las vacas A1A1 - semen sexado; 4) doble de la tasa de reposición de las vacas A1A1 y A1A2 - semen sexado. En todos ellos se utilizan exclusivamente dosis de toros A2A2 y se realiza la reposición con novillas A2A2; sólo cuando la disponibilidad de novillas A2A2 es insuficiente se utilizan novillas A1A2 para la reposición. Los resultados corresponden a la tasa de reposición media estimada en las explotaciones analizadas (38\% anual), un intervalo entre partos de 420 días, una tasa de descarte durante la recría de ter- 
Tabla 8. Valores genómicos medios de los caracteres morfológicos y de los índices sintéticos de tipo para los distintos genotipos de $\beta$-caseína $(n=1868)$.

Table 8. Mean genomic values for morphological traits and composite indexes for $\beta$-casein genotypes $(n=1868)$.

\begin{tabular}{|c|c|c|c|c|c|}
\hline \multirow{2}{*}{ Carácter ${ }^{1}$} & \multicolumn{3}{|c|}{ Genotipo } & \multirow{2}{*}{\multicolumn{2}{|c|}{ Significación }} \\
\hline & $\mathrm{A} 1 \mathrm{~A} 1$ & $\mathrm{~A} 1 \mathrm{~A} 2$ & $\mathrm{~A} 2 \mathrm{~A} 2$ & & \\
\hline EST & 1,093 & 1,023 & 1,029 & 0,254 & \\
\hline ANPE & 0,443 & 0,403 & 0,393 & 0,476 & \\
\hline PC & 0,330 & 0,344 & 0,391 & 0,327 & \\
\hline ANCG & 0,452 & 0,461 & 0,532 & 0,124 & \\
\hline ANGG & $0,270^{a}$ & $0,172^{\mathrm{ab}}$ & $0,069^{b}$ & 0,002 & $* *$ \\
\hline ANGU & 0,900 & 0,892 & 0,908 & 0,855 & \\
\hline VLP & $0,087^{a}$ & $0,224^{b}$ & $0,343^{c}$ & $<0,0001$ & $* * *$ \\
\hline VPP & $0,655^{a}$ & $0,532^{b}$ & $0,454^{b}$ & $<0,0001$ & $* * *$ \\
\hline$A P$ & $0,590^{\mathrm{a}}$ & $0,485^{b}$ & $0,447^{b}$ & $<0,001$ & $* * *$ \\
\hline MA & $1,035^{a}$ & $0,923^{b}$ & $0,847^{c}$ & $<0,0001$ & $* * *$ \\
\hline IAN & 1,259 & 1,227 & 1,167 & 0,083 & \\
\hline AIP & $1,305^{a}$ & $1,185^{b}$ & $1,073^{c}$ & $<0,0001$ & $* * *$ \\
\hline LIG & $1,055^{a}$ & $0,989^{a b}$ & $0,961^{b}$ & 0,020 & * \\
\hline PU & $1,323^{a}$ & $1,267^{a b}$ & $1,187^{b}$ & 0,039 & * \\
\hline CPA & 0,737 & 0,762 & 0,813 & 0,126 & \\
\hline CPP & 0,704 & 0,680 & 0,710 & 0,639 & \\
\hline LPA & $-0,033^{a}$ & $-0,148^{a}$ & $-0,281^{b}$ & $<0,001$ & $* * *$ \\
\hline MOV & $0,970^{a}$ & $0,860^{b}$ & $0,799^{c}$ & $<0,0001$ & $* * *$ \\
\hline ICOt & $599,4^{a}$ & $545,1^{b}$ & $494,5^{c}$ & $<0,0001$ & $* * *$ \\
\hline ICAP & 0,579 & 0,563 & 0,595 & 0,671 & \\
\hline IPP & $0,874^{a}$ & $0,732^{b}$ & $0,647^{c}$ & $<0,0001$ & $* * *$ \\
\hline ICU & $1,50^{\mathrm{a}}$ & $1,44^{\mathrm{ab}}$ & $1,38^{b}$ & 0,019 & $*$ \\
\hline IGT & $1,42^{\mathrm{a}}$ & $1,34^{\mathrm{ab}}$ & $1,28^{b}$ & 0,004 & $* *$ \\
\hline
\end{tabular}

${ }^{1}$ Ver el significado de las abreviaturas de los caracteres en la Tabla 2.

Distintas letras entre columnas indican diferencias significativas $(p<0,05)$. 


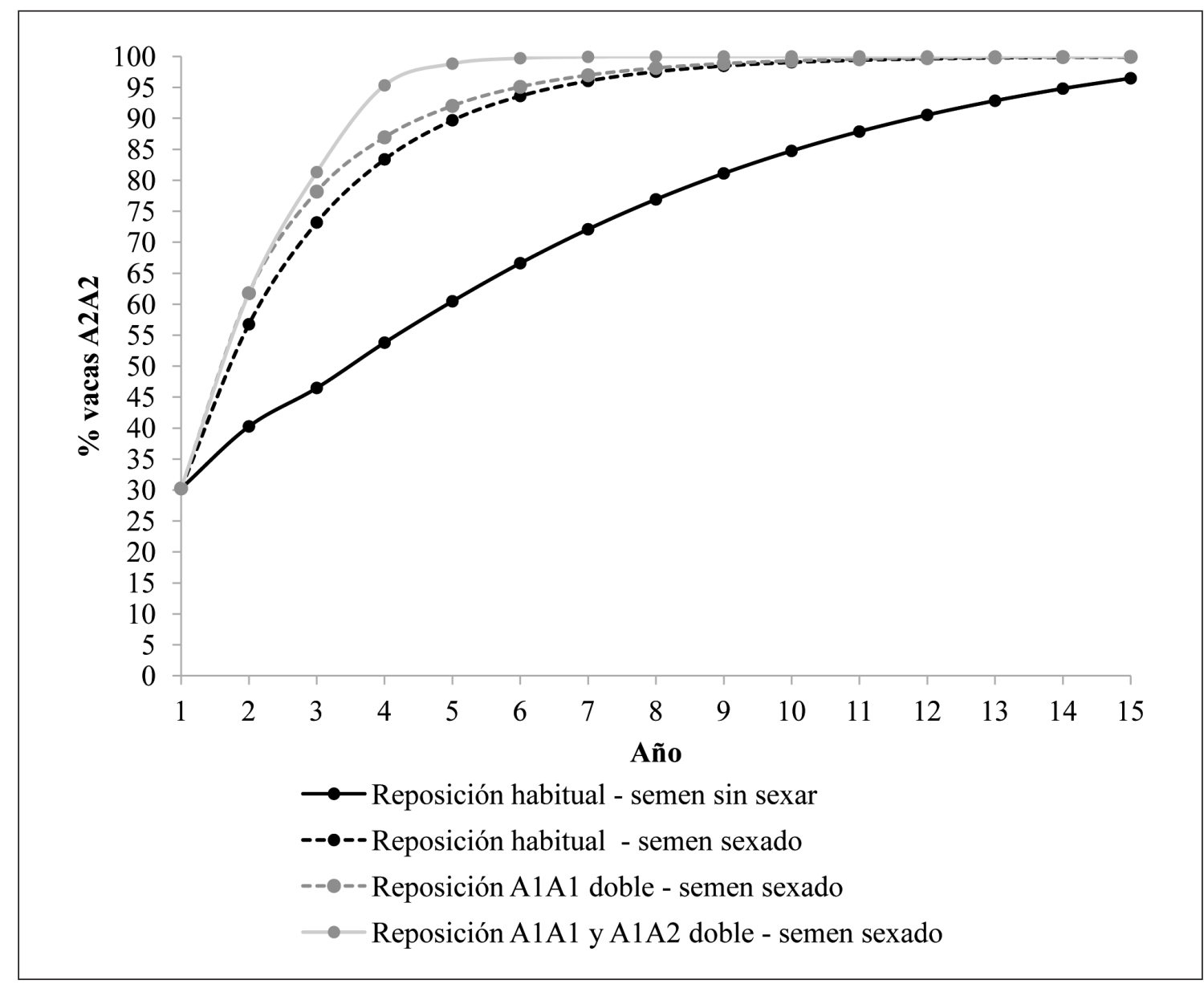

Figura 1. Evolución del porcentaje de vacas A2A2 en los distintos escenarios de conversión a rebaños A2A2 analizados (frecuencia inicial de $A 2=0,55$; reposición anual $=38 \%$ ).

Figure 1. Evolution of A2A2 cows percentages in different conversion strategies in a herd (initial A2 frequency $=0.55$; annual replacement rate $=38 \%$ ).

neras y novillas del $10 \%$, y la frecuencia estimada del alelo que codifica la proteína $\mathrm{A} 2$ $(0,55)$. Hay que tener en cuenta que el periodo de conversión arranca en el momento en que se toma la decisión de transformar el rebaño, por lo que las primeras vacas obtenidas con semen A2A2 entrarán en lactación dos años después.

La duración del periodo de conversión viene limitada por el número de vacas que se debe reponer, voluntaria e involuntariamente, y la disponibilidad de novillas A2A2. Se puede observar que utilizando semen sexado y manteniendo la actual tasa de reposición, es decir sin necesidad de aumentar los costes de reposición, se puede alcanzar la reconversión a un rebaño A2A2 en unos 8 años (pues el proceso de conversión se da por finalizado cuando el porcentaje de vacas A2A2 era superior al $97 \%$ ). Si además del uso de semen 
sexado, se dobla la tasa de reposición de las vacas A1A1 y A1A2 la reconversión se podría hacer en unos 5 años. Si no se utilizara semen sexado ni se aumentara la tasa de reposición, el periodo se extendería hasta los 15 años. Aumentar la reposición exclusivamente de las vacas A1A1 no aporta mucho al proceso de conversión, dado que la reposición voluntaria afecta a un escaso número de vacas.
Si se parte de una situación más desfavorable, frecuencia de $A 2=0,35$, aunque aumenta la duración del periodo de conversión, la comparación entre escenarios es similar (Figura 2). Incluso con esa menor frecuencia, se podría terminar la conversión en un plazo de 9 años usando semen sexado de toros A2A2 sin necesidad de aumentar la tasa de reposición de las vacas.

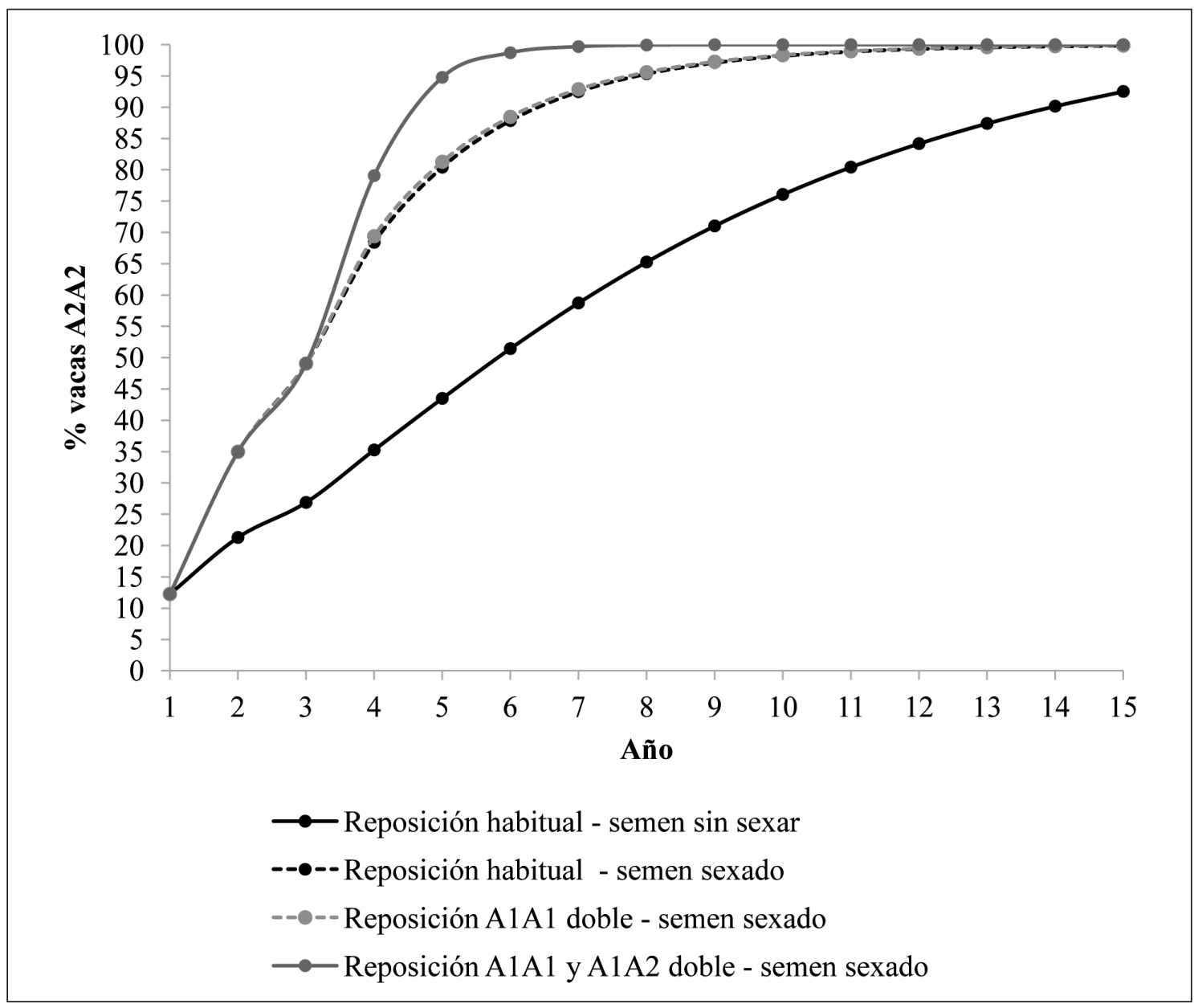

Figura 2. Evolución del porcentaje de vacas $A 2 A 2$ en los distintos escenarios de conversión a rebaños A2A2 analizados (frecuencia inicial de $A 2=0,35$; reposición anual $=38 \%$ ).

Figure 2. Evolution of A2A2 cows percentages in different conversion strategies in a herd (initial A2 frequency $=0.35$; annual replacement rate $=38 \%$ ). 
La Figura 3 recoge la evolución de la población bajo distintas tasas de reposición involuntaria de las vacas, cuando se parte de una situación en la que se utiliza semen sexado. Como cabía esperar aquellas explotaciones que se encuentran por debajo de la tasa media de reposición de las explotaciones analizadas $(38 \%)$ van a precisar más años para la conversión a leche A2. Sin embargo, la conversión no se alcanzará mucho más rápidamente en las explotaciones que realizan una reposición más elevada (50\%, Figura 3), dado que un alto porcentaje de reposición implica que inicialmente no haya suficientes noviIlas A2A2 para hacer toda la reposición, lo que obliga a utilizar novillas A1A2.

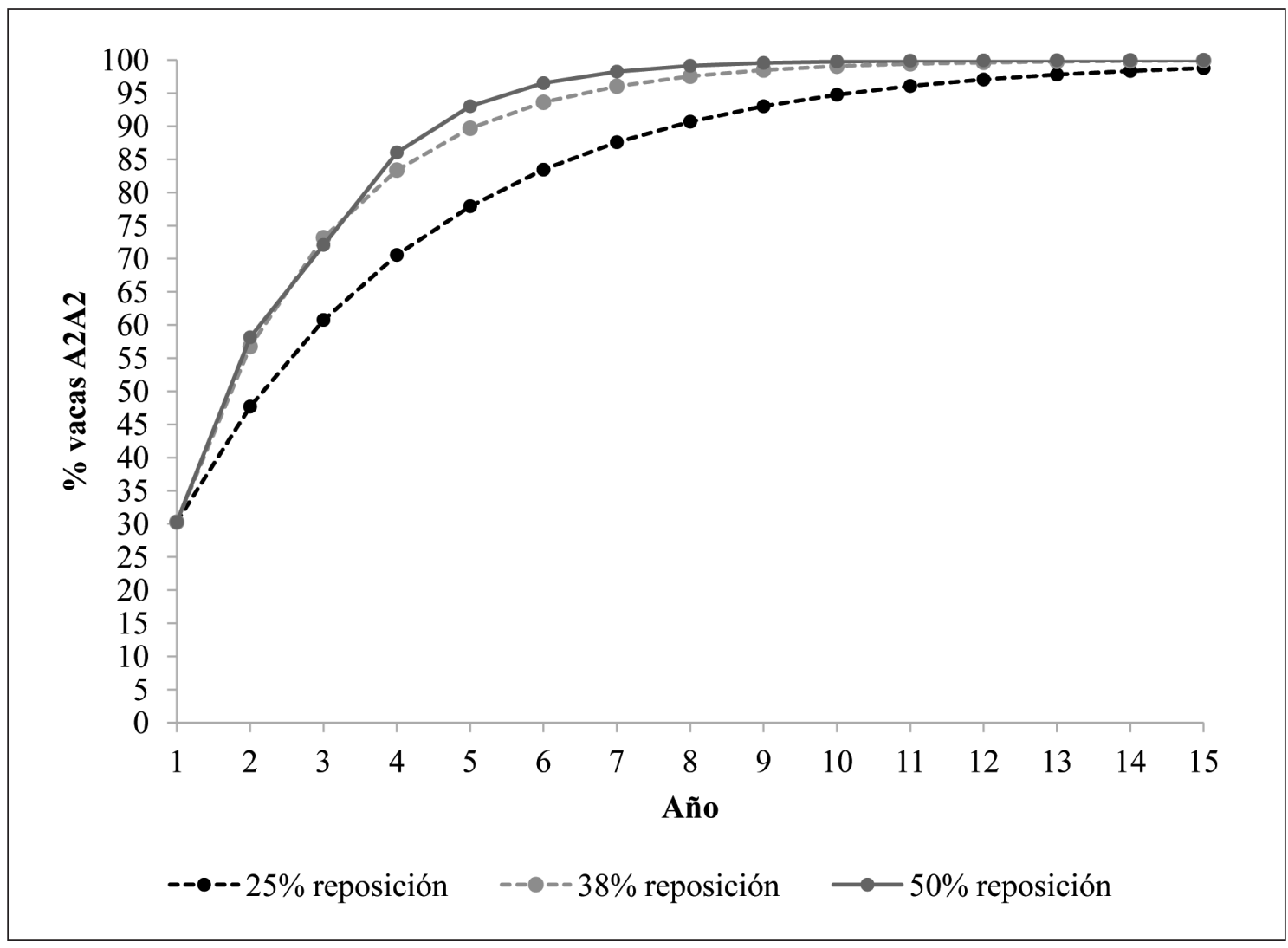

Figura 3. Evolución del porcentaje de vacas A2A2 bajo distintas tasas de reposición involuntaria de vacas (frecuencia inicial de $A 2=0,55$; empleo de semen sexado).

Figure 3. Evolution of A2A2 cows percentages under different involuntary replacement rates of cows (initial A2 frequency $=0.55$; use of sexed semen). 
Estos resultados son semejantes a los obtenidos en los trabajos de Mencarini et al. (2013) y Gödert et al. (2017). Los escenarios simulados y los valores de los parámetros tenidos en cuenta varían respecto a los considerados en este trabajo, variando los resultados alcanzados, pero llegando a conclusiones similares: la mejor estrategia para hacer una rápida conversión es el uso de semen sexado, y solo si se tiene la posibilidad de recibir inmediatamente un pago extra por la leche A2 parece razonable aumentar la tasa voluntaria de reposición.

Como la conversión a leche A2 implica la utilización exclusiva de toros homozigotos $A 2$, se puede pensar en un posible retraso genético respecto al uso del conjunto de toros disponibles. Sin embargo, los resultados recogidos en la Tabla 9 no indican que se vaya a producir retraso genético en ninguno de los caracteres con valoración genética analizados, puesto que no parece haber diferencias desfavorables entre los toros homozigotos $A 2$ y el resto. Los toros en prueba A2A2 presentan un mayor valor genómico para kilogramos de leche y proteína que el resto de toros, coincidiendo con lo observado en novillas (Tabla 5), aunque de forma no esperada también presentan mayor valor genómico para kilogramos de grasa, sin presentar diferencias significativas en porcentaje de grasa.

Pese a que no cabe esperar que el uso exclusivo de toros A2A2 afecte directamente al valor genético medio de las explotaciones, habrá que tener en cuenta que el número efectivo de toros se reduce de forma significativa (un $60 \%$ ), lo que unido a las posibles asociaciones con genes o haplotipos deletéreos (como la encontrada en este trabajo con HDC) obligará a tener especialmente en cuenta el parentesco entre animales en el diseño de apareamientos. Además, si la conversión se produjera en un número importante de explotaciones en control lechero oficial, también se debería tener en cuenta que a nivel de programa de me- jora la presión de selección sobre los machos disminuirá, afectando negativamente a su progreso genético.

Por último, la Tabla 10 recoge el coste del proceso de conversión a leche A2 para las cuatro estrategias analizadas y dos frecuencias iniciales para el alelo que codifica la proteína A2. La estrategia más económica es hacer una reposición preferente con novillas A2A2 sin realizar ningún cambio en el manejo actual de las explotaciones, pero el largo periodo de conversión le hace perder interés, y para alcanzar la conversión en un periodo razonable hay que pensar en la utilización de semen sexado. Utilizando semen sexado, aumentar voluntariamente la tasa de reposición de las vacas A1A1 encarece mucho la conversión sin presentar muchas ventajas en el tiempo empleado en ella. El tiempo sí que se acorta relevantemente cuando también se aumenta voluntariamente la reposición de las vacas $A 1 A 2$, pero el aumento en los costes obliga a desconsiderar esta estrategia a no ser que los tres años que permite acortar el periodo de conversión sean especialmente importantes por la inminencia de cambios en el mercado de la leche. En conclusión, parece que la estrategia más razonable de conversión a rebaños $\mathrm{A} 2 \mathrm{~A} 2$ a corto plazo ante la posibilidad de que aparezcan nuevas demandas de mercado, es la utilización de semen sexado de toros homozigotos A2 manteniendo su tasa de reposición.

No obstante, el interés de la utilización de semen sexado viene determinado por la tasa de reposición involuntaria de los rebaños. Como ya se ha indicado el proceso de conversión viene limitado por la relación entre el número de vacas a reponer anualmente y la disponibilidad de novillas A2A2. Para tasas de reposición involuntaria del $50 \%$ se hace obligatorio el uso de semen sexado, pues de lo contrario no hay suficiente disponibilidad de novillas A2A2 para hacer la reposición anual (Tabla 11). Por contra, para tasas de reposi- 
Tabla 9. Valores genómicos medios de toros probados y genómicos disponibles para el genotipo A2A2 y el resto de genotipos para $\beta$-caseína.

Table 9. Mean genomic values of proven and genomic bulls available for the A2A2 and the other genotypes for $\beta$-casein.

\begin{tabular}{|c|c|c|c|c|c|}
\hline \multirow{2}{*}{ Toros } & \multirow{2}{*}{ Carácter ${ }^{1}$} & \multicolumn{2}{|c|}{ Genotipo } & \multirow{2}{*}{\multicolumn{2}{|c|}{ Significación }} \\
\hline & & $\mathrm{A} 2 \mathrm{~A} 2$ & $\mathrm{~A} 1 \mathrm{~A} 2$ y $\mathrm{A} 1 \mathrm{~A} 1$ & & \\
\hline \multirow[t]{13}{*}{ Probados } & & $(n=17)$ & $(n=73)$ & & \\
\hline & $\mathrm{KL}$ & 683,94 & 860,60 & 0,155 & \\
\hline & $\% G$ & $-0,019$ & $-0,079$ & 0,228 & \\
\hline & KG & 22,29 & 22,07 & 0,967 & \\
\hline & $\% \mathrm{P}$ & 0,004 & $-0,032$ & 0,161 & \\
\hline & KP & 22,65 & 24,40 & 0,639 & \\
\hline & ICO & 2571,06 & 2684,99 & 0,357 & \\
\hline & RCS & 102,94 & 103,75 & 0,692 & \\
\hline & LON & 107,41 & 105,95 & 0,519 & \\
\hline & ICAP & 0,66 & 0,58 & 0,717 & \\
\hline & IPP & 0,59 & 0,72 & 0,502 & \\
\hline & ICU & 1,20 & 1,23 & 0,873 & \\
\hline & IGT & 1,17 & 1,21 & 0,781 & \\
\hline \multirow[t]{13}{*}{ Genómicos } & & $(n=33)$ & $(n=42)$ & & \\
\hline & $\mathrm{KL}$ & 1356,33 & 1110,52 & 0,030 & * \\
\hline & $\% \mathrm{G}$ & 0,067 & 0,059 & 0,873 & \\
\hline & KG & 55,45 & 45,43 & 0,019 & * \\
\hline & $\% \mathrm{P}$ & 0,046 & 0,036 & 0,593 & \\
\hline & KP & 48,67 & 39,48 & 0,003 & ** \\
\hline & ICO & 3959,82 & 3638,12 & 0,002 & ** \\
\hline & RCS & 114,24 & 112,74 & 0,444 & \\
\hline & LON & 123,18 & 120,12 & 0,065 & \\
\hline & ICAP & 0,92 & 0,86 & 0,697 & \\
\hline & IPP & 1,22 & 1,19 & 0,823 & \\
\hline & ICU & 2,19 & 2,12 & 0,535 & \\
\hline & IGT & 2,09 & 1,98 & 0,408 & \\
\hline
\end{tabular}

${ }^{1}$ Ver el significado de las abreviaturas de los caracteres en la Tabla 2. 


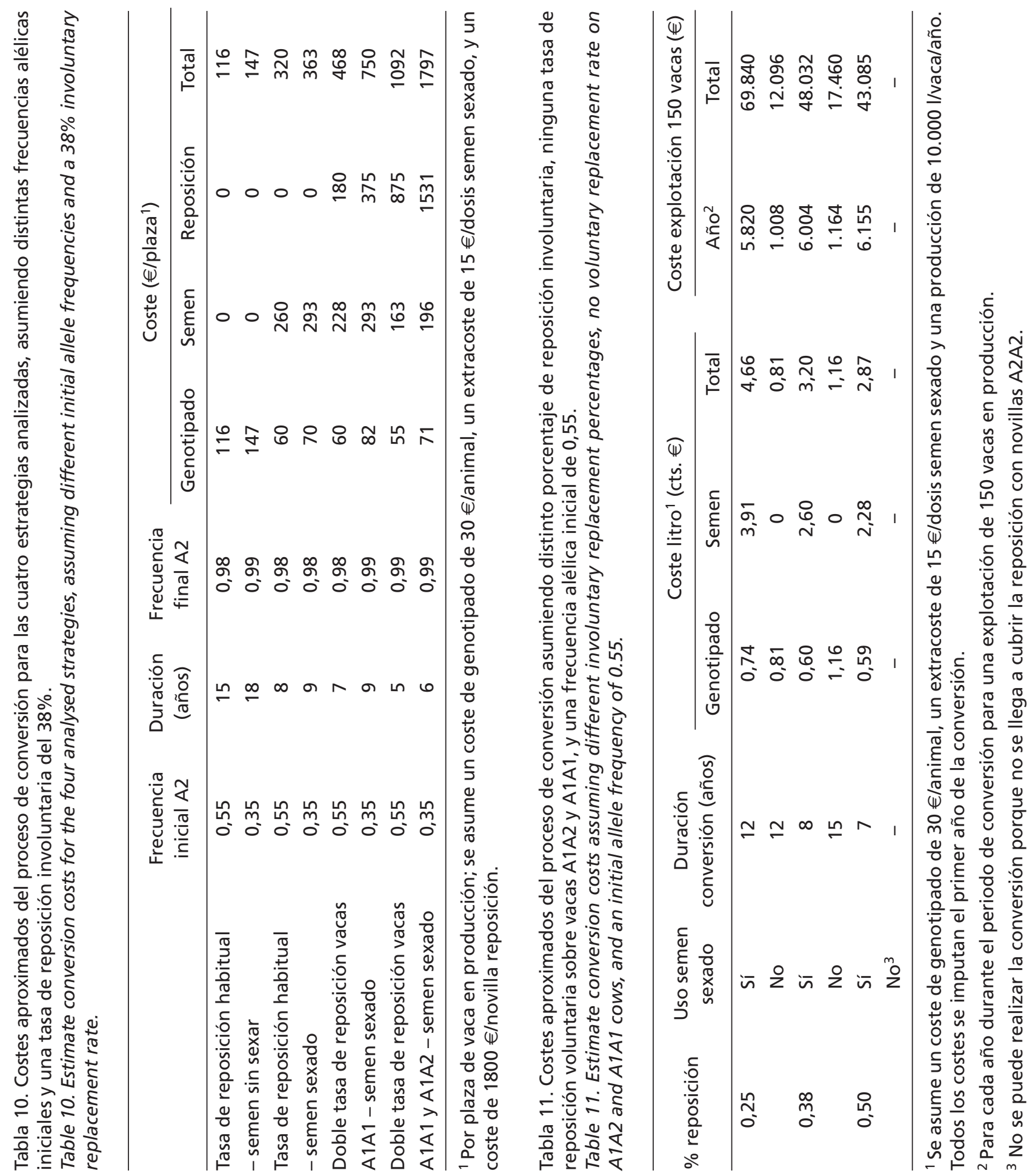


ción inferiores, del $25 \%$, no se justifica la utilización de semen sexado pues aumenta los costes de la explotación innecesariamente dado que la reposición con novillas A2A2 se puede cubrir, partiendo de una frecuencia de 0,55 del alelo $A 2$, sin su utilización (Tabla 11).

Con el coste actual de las dosis de semen sexado, el coste estimado de la conversión es muy elevado, sobre 3-4 céntimos de euro más por litro de leche, y no se justifica si todavía no existe un pago adicional de la leche A2 y lo único que se persigue con la conversión es posicionarse adecuadamente para una posible demanda de mercado. Gödert et al. (2017) llegan a resultados semejantes, con costes también muy elevados utilizando semen sexado, que les llevan a concluir que, sin un aumento asegurado de los ingresos, la selección a favor del genotipo A2A2 debería supeditarse a otros objetivos de mejora en la mayoría de las explotaciones alemanas.

Hay que indicar que la evaluación económica realizada es simplemente orientativa del coste máximo que podría suponer la conversión a una explotación tipo que en la actualidad no realiza evaluación genómica de novillas ni utiliza semen sexado, y debería de ajustarse a la realidad de cada explotación antes de valorar su interés particular de la conversión a leche A2. De hecho, en las explotaciones analizadas el genotipado se viene realizando por razones ajenas al genotipo para $\beta$-caseína como son las ventajas de la selección genómica en novillas, la confirmación de paternidades y el diseño de apareamientos, por lo que el coste de genotipado no debería de imputarse. Del mismo modo, algunas explotaciones ya utilizan parcialmente semen sexado para responder a sus necesidades de reposición, por lo que en este caso tampoco se debería imputar el sobrecoste del sexado de semen. Además, tampoco es necesario utilizar siempre semen sexado en todas las vacas, y es de esperar el extracoste sobre el semen no sexado disminuya los próximos años.
Finalizando, no está claro que a corto plazo vaya a surgir una demanda real por parte de los consumidores españoles de leche $A 2$, sino más bien cabe pensar que a los consumidores les van a surgir ofertas de leche A2 por parte de algunos productores en busca de un desacoplamiento de los precios del mercado de la leche. A medio plazo, aquellas ganaderías mejor posicionadas para realizar el proceso de conversión a leche $A 2$ serían las que además de presentar una mayor frecuencia del alelo que codifica para la $\beta$-caseína tipo $A 2$, presentaran una menor tasa de reposición involuntaria de los animales, lo cual les permitiría reducir los costes asociados al uso de semen sexado que en determinadas situaciones es necesario para alcanzar la conversión de un rebaño a la producción de leche A2 en un periodo razonable.

\section{Conclusiones}

El grupo de polimorfismos que codifica la $\beta$ caseína tipo A2 es predominante en las explotaciones analizadas sobre el grupo que codifica la $\beta$-caseína tipo A1. No existen importantes asociaciones desfavorables entre el alelo que codifica la $\beta$-caseína tipo A2 y el valor genético para caracteres productivos, funcionales y morfológicos, por lo que no cabría esperar una disminución importante del nivel genético de las explotaciones si se optara por convertir los rebaños a la producción de leche A2.

La conversión de los rebaños a producción de leche $\mathrm{A} 2$ basada en la utilización exclusiva de machos de genotipo A2A2 no tiene por qué producir un retraso genético en las explotaciones analizadas, dado que su nivel genético no se ha encontrado que sea inferior al de los toros de los otros genotipos en el caso del centro de inseminación artificial analizado. No obstante, se reduce significativamente el número de toros disponibles, por lo que ha- 
bría que tener especialmente en cuenta su información sobre genes recesivos y parentesco en el diseño de apareamientos, así como la implicación sobre el progreso genético si las explotaciones están integradas en un programa de mejora genética.

Además de la frecuencia inicial de los polimorfismos que codifican la $\beta$-caseína tipo A2, la tasa de reposición involuntaria de los rebaños condiciona de forma importante la duración y el coste del proceso de conversión a leche A2. El uso de semen sexado permite acortar la duración de la conversión, especialmente cuando la tasa de reposición involuntaria es elevada, pero atendiendo a los costes actuales del semen sexado la encarece de forma importante, por lo que su empleo sistemático únicamente parece recomendable si existe la posibilidad inmediata de recibir un pago extra por la leche A2. En este contexto, las explotaciones con una mayor tasa de reposición estarán mejor posicionadas.

Si la conversión a la producción de leche A2 no responde a una necesidad inmediata de desacoplarse de los precios de mercado, pero sí estar preparados a medio plazo ante una posible demanda de la industria asegurando la recogida y venta de la leche producida, el único cambio razonable a introducir en las explotaciones es la utilización de toros A2A2. $\mathrm{Ni}$ el uso de semen sexado, ni aumentar voluntariamente la reposición se justifica desde el punto de vista económico. En este contexto, al contrario que en el anterior, las explotaciones mejor posicionadas serán aquellas con menor tasa de reposición de vacas.

No ha sido objeto de este trabajo abordar las implicaciones de la leche A2 sobre la salud humana ni las dificultades que puede presentar su comercialización, pero una vez analizadas las posibilidades e implicaciones de la conversión de las explotaciones a la producción exclusiva de ese tipo de leche ambos aspectos deberían ser analizados con mayor profundidad.

\section{Agradecimientos}

Este trabajo forma parte de la colaboración de los autores con la Asociación GIFE (Gipuzkoako Frisoi Elkartea) para la gestión de los resultados de genotipado de novillas.

\section{Referencias bibliográficas}

Asledottir T, Le TT, Petrat-Melin B, Devold TG, Larsen LB, Vegarud GE (2017). Identification of bioactive peptides and quantification of $\beta$-casomorphin-7 from bovine $\beta$-casein A1, A2 and I after ex vivo gastrointestinal digestion. International Dairy Journal 71: 98-106.

Babidge, G (2017). CEO's Report. In: The a2 Milk Company Ltd. Annual Report 2016-2017.

Brooke-Taylor S, Dwyer K, Woodford K, Kost N (2017) Systematic review of the gastrointestinal effects of A1 compared with A2 $\beta$-Casein. Advances in Nutrition 8: 739-748.

Frisona Española (2017). Proyecto genómico en Gipúzcoa. Frisona Española 218: 40-46.

Gödert M, Brandt H, Erhardt G (2017). Background of beta-Casein A2 in bovine milk - strategies and limitations by breeding and the dairy industry with regard to a possible new demand (en alemán). Züchtungskunde 89 (6) S: 451-474.

Hallén E, Allmere T, Näslund J, Andrén A, Lundén A (2007). Effect of genetic polymorphism of milk proteins on rheology of chymosin-induced milk gels. International Dairy Journal 17: 791-799.

He M, Jianqin S, Jiang ZQ, Yang YX (2017). Effects of cow's milk beta-casein variants on symptoms of milk intolerance in Chinese adults: a multicentre, randomised controlled study. Nutrition Journal 16:72.

Ho S, Woodford K, Kukuljan S, Pal S (2014). Comparative effects of A1 versus A2 beta-casein on gastrointestinal measures: a blinded randomised cross-over pilot study. European Journal of Clinical Nutrition 68: 994-1000.

Jianqin S, Leiming X, Lu X, Yelland GW, Ni J, Clarke AJ (2016). Effects of milk containing only A2 
beta casein versus milk containing both $\mathrm{A} 1$ and A2 beta casein proteins on gastrointestinal physiology, symptoms of discomfort, and cognitive behavior of people with self-reported intolerance to traditional cows' milk. Nutrition Journal, 15: 35.

Jiménez JA (2015a). Nuevo chip de baja densidad: información adicional al valor genómico. Frisona Española 207: 56-57.

Jiménez JA (2015b). La genómica ayuda a detectar problemas y a evitarlos. Frisona Española 208: 42-43.

Kamiński S, Cieślińska S, Kostyra E (2007). Polymorphism of bovine beta-casein and its potential effect on human health. Journal of Applied Genetics 48(3): 189-198.

Lin CY, McAllister AJ, Ng-Kwai-Hang KF, Hayes JF, Batra TR, Lee AJ, Roy GL, Vesely JA, Wauthy JM, Winter KA (1989). Relationships of Milk Protein Types to Lifetime Performance. Journal of Dairy Science 72: 3085-3090.

Massella E, Piva S, Giacometti F, Liuzzo G, Zambrini AV, Serraino A (2017). Evaluation of bovine beta casein polymorphism in two dairy farms located in northern Italy. Italian Journal of Food Safety 6(3): 6904.

Mencarini IR, Woodford KB, Old KM (2013). Comparing herd selection strategies for $\mathrm{A} 2$ beta-casein. Proceedings of the New Zaeland Society of Animal Production Vol 73: 149-154.

Morris CA, Hickey SM, Cullen NG, Prosser CG, Anderson RM, Tate ML (2005). Associations between $\beta$ casein genotype and milk yield and composition in grazing dairy cows. New Zealand Journal of Agricultural Research 48(4): 441-450.

Ng-Kwai-Hang KF, Hayes JF, Moxley JE, Monardes HG (1984). Association of genetic variants of ca- sein and milk serum proteins with milk, fat and protein production by dairy cattle. Journal of Dairy Science 67: 835-840.

Ojala M, Famula TR, Medrano JF (1996). Effects of milk protein genotypes on the variation for milk production traits of Holstein and Jersey cows in California. Journal of Dairy Science 80(8): 1776-1785.

Olenski K, Kamiński S, Szyda J, Cieslinska A (2010). Polymorphism of the beta-casein gene and its associations with breeding value for production traits of Holstein-Friesian bulls. Livestock Science 131: 137-140.

Potočnik K, Luštrek B, Kaić A (2016). Does the selection on ss-casein affect the traits important for dairy production of slovenian brown swiss cattle? Acta agriculturae Slovenica, Supplement 5: 89-93.

Tejedor MT (1986). Polimorfismo genético de las proteínas lácteas en ganado vacuno lechero. Estudio de las asociaciones haplotípicas y su relación con la respuesta a la selección. Tesis Doctoral. Universidad de Zaragoza.

Truswell AS (2005). The A2 milk case: a critical review. European Journal of Clinical Nutrition 59: 623-631.

Van Eenennaam AL, Medrano JF (1991) Milk protein polymorphisms in California dairy cattle. Journal of Dairy Science 74: 1730-1742.

Velmala R, Vilkki J, Elo K, Mäki-Tanila A (1995). Casein haplotypes and their association with milk production traits in the Finnish Ayrshire cattle. Animal Genetics 26: 419-425.

(Aceptado para publicación el 2 de enero de 2019) 